\title{
Improving Access to Preventive Services for Marginalized Families During Early Childhood: An Integrative Review of Inter-organizational Integration Interventions
}

\author{
Dawn Smith*, Wendy E. Peterson, Maria Jaglarz and Kelly Doell
}

\author{
School of Nursing, Faculty of Health Sciences, University of Ottawa, 451 Smyth Road, Room 3251B, Ottawa, ON, K1H \\ 8M5, Canada
}

\begin{abstract}
Marginalized populations exhibit low rates of preventive service use, often avoiding use of non-urgent services. Poor access to preventive and health promoting care serves to maintain inequities in health experienced by many marginalized populations. Of particular concern are marginalized families with young children below the age of school entry, when physical, emotional and psychological foundations for life-long health are being established. Many community based organizations recognize the need to improve families' access and use of preventive services. However, they are faced with a gap in understanding what inter-organizational interventions could be implemented to improve integration of services particularly focused on addressing experiences of marginalized families. Therefore, we used the integrative review method to identify and describe inter-organizational (I-O) interventions in the literature that aim to improve access to preventive services by marginalized families. As per integrative review methods, the literature was searched for research studies using qualitative, quantitative or mixed method designs, and investigating I-O interventions aiming to improve access to preventive services through increased service integration. Three levels of screening and relevance review identified fourteen articles. A conceptual model informed by socio-ecological theory was used to classify interventions as relational or structural. Results show that reports of rigorously conducted studies of I-O interventions are relatively sparse, and emphasize structural factors such as shared leadership, shared review or development of policies/protocols, changes to referral mechanisms and geographical/caseload matching. Interorganizational interventions that influence relational factors were rare but have included: joint training/education, facilitated communication, addition of an integration role, and strategic partnerships. We suggest that combining both structural- and relational-focused strategies in inter-organizational integration intervention design may have greater impact on improving access to preventive services for marginalized families, with increased use of early childhood preventive services contributing to reducing health disparities.
\end{abstract}

\section{INTRODUCTION}

There is strong evidence that early childhood is a crucial time when preventive services can optimize critical periods of brain development and contribute significantly to life-long health $[1,2]$. Preventive services for families during the early childhood period are often provided by a range of agencies and include services such as: regular and sustained care by a primary care giver such as physician or nurse practitioner, prenatal education, post partum preventive care, breastfeeding support, immunization and well child development services, literacy education, and recreation and social interventions such as play groups. Timely access to, and participation in these services can improve key determinants of child health including breastfeeding initiation and duration rates, maternal attachment and health behaviors and coping skills among new parents [3-6]. This is important for the children of marginalized families, who are particularly susceptible to poorer health outcomes due to factors that influence their families' access to healthcare

*Address correspondence to this author at the School of Nursing, Faculty of Health Sciences, University of Ottawa, 451 Smyth Road, Room 3251B, Ottawa, ON K1H 8M5, Canada; Tel: 613-562-5800, Ext. 8420;

Fax: 613-562-5443; E-mail: dsmith@uottawa.ca services such as lower socio-economic status, language barriers, and disability.

Agencies with specialized service delivery mandates have had success in creating the environment and relationships that encourage higher rates of service access and use by marginalized families (e.g. specialized services for pregnant teens, new immigrants or Aboriginal families) [7]. Several studies have shown success providing a range of services to small proportions of eligible marginalized families within resource intensive integrated service delivery models [8-10]. However, marginalized families continue to be infrequent users of preventive services that are more widely available through a variety of community based organizations (e.g. public health units, community health centers), and are overrepresented among families whose children begin school with poor physical, emotional and language skills [11]. This infrequent service use among marginalized families occurs despite referrals between agencies providing preventive services through public health and various community-based organizations [12, 13]. Early evaluation reports of initiatives such as Sure Start in the United Kingdom [14], and Strengthening Families in New Zealand [15] have recognized the complexity of improving integration across agencies, and identified requirements such as the strong local management and shared governance 
structures, resource intensiveness of inter-agency collaboration, sophisticated IT systems, a common assessment framework, and shared and clarified language. Clearly, the need for evidence-based inter-organizational interventions that make existing preventive and health promoting services more widely accessible to marginalized families is urgent. Further, theory and knowledge of interventions to improve the logic underpinning interorganizational integration efforts is important to reducing inequities in access to preventive services.

This paper reports the findings of an integrative review of the literature examining system interventions that aim to improve inter-organizational (I-O) integration for the purpose of improving access and use of non-urgent and health promoting preventive services (hereafter referred to as preventive services) by marginalized families, a topic that has not to our knowledge been previously examined in the literature. Though definitions vary widely, there is general agreement that integration is an ongoing process that involves multiple partners, combines resources and incorporates notions of comprehensiveness, co-operation and coordination $[16,17]$. We define integration as the process "...whereby different services that are usually autonomous organizations, work together for specific community residents to improve health and social care" [18]. We describe the theoretical perspective and methods used to search, screen, appraise and critically review the literature. Results of the 14 included studies are described and implications for research, policy and system change are outlined. The findings from this review have relevance for decision makers concerned with improving access and participation of marginalized populations in preventive services that are delivered through multiple agencies, programs and provider disciplines.

\section{THEORETICAL PERSPECTIVE}

We developed a conceptual model informed by socioecological theory and concepts of service integration [18] to identify and classify I-O system integration interventions intended to improve access to services for marginalized families. Socio-ecological theory recognizes that peoples' development and well being are influenced by their interactions with multiple layers and dimensions of their environments [19]. A socio-ecological perspective facilitates analysis of how social, historical and cultural factors interact at multiple levels to explain marginalized populations' patterns of service access and use. This ties in closely with common definitions of integrated care as requiring strategies employed at multiple levels (e.g. policy, organizational, individual) in efforts to harmonize services [20]. Multiple intervention planning based on socio-ecological theory involves strategically selecting interventions targeted at multiple levels of the system to achieve more equitable, efficient and/or effective outcomes [21]. For example, integration interventions can aim to harmonize services between organizations, programs within one organization and collaboration between individual providers. Specific interventions may aim to provide continuity of care for clients by targeting administrative, informational and relational aspects of care [22].
Clients' experiences of service integration are significantly influenced by factors in two dimensions: structural and interpersonal $[16,18]$. Structural factors are system or agency characteristics "...that smooth the progress of, or restrain collaborative efforts" [16]. Interpersonal factors are defined as "...the characteristics of relationships between individuals that enhance or inhibit collaborative efforts" [16], which for our purposes we termed 'relational'.

This review focuses on organizational interventions to improve access to care as experienced by marginalized families. In the literature, marginalized populations are often described as 'vulnerable' or 'hard to reach' and include for example, refugees [23], working poor families [24], disadvantaged mothers [25], adolescent mothers [26] and those living with mental illness or intellectual disabilities [27]. While to be "vulnerable" often means to be particularly susceptible and at risk for poor physical, psychological, or social health, the term marginalization expresses a critique of the societal structures that peripheralize individuals and groups from a dominant, central majority on the basis of their identities, associations, experiences, and environments $[28,29]$. Use of the term marginalization emphasizes that it is the responsibility of the health and social system (and not only individual organizations) to improve access to preventive services for populations with diverse identities and experiences through improved system integration.

Agency efforts to improve families' access to preventive services most commonly address individual-level structural and practical barriers such as transportation and childcare. For example, bus tickets and on-site day care are commonly available for families using agency-specific services. Asthana and Halliday [10] identified the need for opportunities to encourage dissemination of information between agencies in order to facilitate and improve upon innovated service delivery techniques in their study of reducing disparities between rural and urban funding for health care services. However, the reality for families is that preventive services are often provided by multiple agencies including individual medical practices, public health, community-based social services, publicly funded community health centers, non-governmental organizations, recreation departments and educational institutions. Therefore, the nature of the early childhood preventive service system is often structurally fragmented and difficult to navigate for both providers and clients. While service integration efforts do exist, the emphasis has been predominantly on evaluating improvements on efficiency and effectiveness, rather than reach to families experiencing deeply embedded inequities in health [17]. Difficulties in design and implementation of the complex interventions [30, 31], lack of intensive intervention and research funding, and intervention science capacity among public health and community based agencies [32] has also contributed to this gap in the literature.

Further, clients' negative experiences or perceptions of services may deter their access and participation, ultimately adversely influencing health and developmental outcomes of children and perpetuating deeply embedded inequities in health among population groups. Research has yet to 
examine how to better integrate services across sectors, organizations and programs to help identify how to reduce these less tangible barriers to care [33]. Studies that have attempted to integrate care by intervening at the interprofessional level have identified the need for synergistic organizational-level interventions [34, 35]. Therefore, identifying these less tangible I-O barriers to integrated preventive care has the potential to improve the health and social safety-net for those that have historically 'fallen through the cracks'. The purpose of this integrative review is to synthesize the literature describing interventions to improve I-O integration. We pay particular attention to interventions aimed at improving I-O system integration for the purpose of improving equity of access to communitybased preventive services for marginalized families.

\section{METHODS}

Health care providers, researchers, and policy makers need the large volume of existing information to be evaluated and integrated to support decision-making. Systematic reviews are used on well researched interventions, and help determine decisions for the particular populations, settings, and treatment variations [36]. In contrast, an integrative review is effective when there is a dearth of evidence in the literature. A wider lens is required to gather information, and may include examination of interventions across population types and settings. Integrative reviews present the state of the literature on a given phenomenon or problem and contribute to theory development $[37,38]$. They focus on examining the depth and breadth of a topic, include experimental and nonexperimental research and permit qualitative findings to also have a presence. We adapted the stages for conducting integrative reviews described by Whittemore \& Knafl [38]: problem identification, literature search, relevance screening, quality appraisal, data analysis, evaluation and extraction.

\section{Problem Identification}

We conducted this integrative review of the literature to determine the state of knowledge about interventions a) targeted at the I-O level of the system and b) intended to improve equity of access to non-urgent community-based health promoting and preventive services among marginalized families during the early childhood period.

\section{Literature Search}

Six databases were searched (MedLine, CINAHL, HealthStar, Embase, PsychInfo, Cochrane Library) using terms from the following categories: marginalized families, systems integration, intervention, health services, and access. The search strategy was adapted and tailored for each database based on their usage of MESH terms or differences in headings used (see Table $\mathbf{1}$ for an example). Given the dynamic context of health and social service systems, the search was limited to articles published between January 2000 and June 2007. While our population of interest is families in the early childhood stage of development, our initial search yielded very few articles.

Therefore, we expanded the term marginalized families, to include marginalized populations, based on the rationale that attending the person's experience of accessing services, rather than a developmental stage would address relational as well as structural aspects of the issue. The reference lists of promising articles were also searched for additional articles not identified by the electronic database search. Selected websites such as Best Start: Ontario's Maternal Newborn and Early Child Development Resource Centre were searched and findings were added to the final set of manuscripts for review. Fig. (1) shows the steps and outcomes of the search and screening strategy. A total of 1503 articles were identified once duplicates were removed.

\section{Screening}

- A set of inclusion and exclusion criteria were created to screen each article generated by the database search. Inclusion criteria were:

- Language: English only.

- Population: Marginalized populations.

- $\quad$ Setting: The study or experience described occurred in health and social care and socioeconomic contexts similar to Canada (e.g. Europe, Australia, Canada or the United States).

Table 1. Search Strategy for MEDLINE Database

\begin{tabular}{|c|c|}
\hline Category of Terms & Examples of Search Terms \\
\hline Marginalized Population & Special population.tw, high risk population.tw, at risk population.tw, vulnerable famil\$.tw, vulnerable population.mp \\
\hline \multicolumn{2}{|r|}{ AND } \\
\hline Systems Integration & Systems integration.mp, systems design.tw., systems analysis.mp, integrated health care system.mp \\
\hline \multicolumn{2}{|r|}{ AND } \\
\hline Intervention & Early childhood intervention.tw, childhood intervention\$.tw, early treatment.tw, inter-institutional relations.mp \\
\hline \multicolumn{2}{|r|}{ AND } \\
\hline Health Services & $\begin{array}{c}\text { Health services administration.tw, primary care.tw, patient-centred care.mp, community health service.mp, child health } \\
\text { services.tw }\end{array}$ \\
\hline \multicolumn{2}{|r|}{ AND } \\
\hline Access & Attitude to health.mp, health personnel attitude.mp, patient satisfaction.mp, access to health care.mp \\
\hline
\end{tabular}


- $\quad$ Purpose: To report research findings from primary research studies that aimed to describe the experience of service integration for the purpose of improving access to services by marginalized populations. Quantitative, qualitative and mixed method designs were included. Quantitative studies and mixed method studies must have included I-O integration as a variable and qualitative studies must have investigated interagency integration and access to services among marginalized populations.

- Type of services: Health promoting or preventive services for marginalized populations.

Exclusion criteria were:

- $\quad$ Theses or dissertations.

- There was no abstract available.

- Articles describing integration other than services (e.g. integration of evidence) or without interventions implemented at the I-O level.

- $\quad$ Literature Reviews and commentaries.

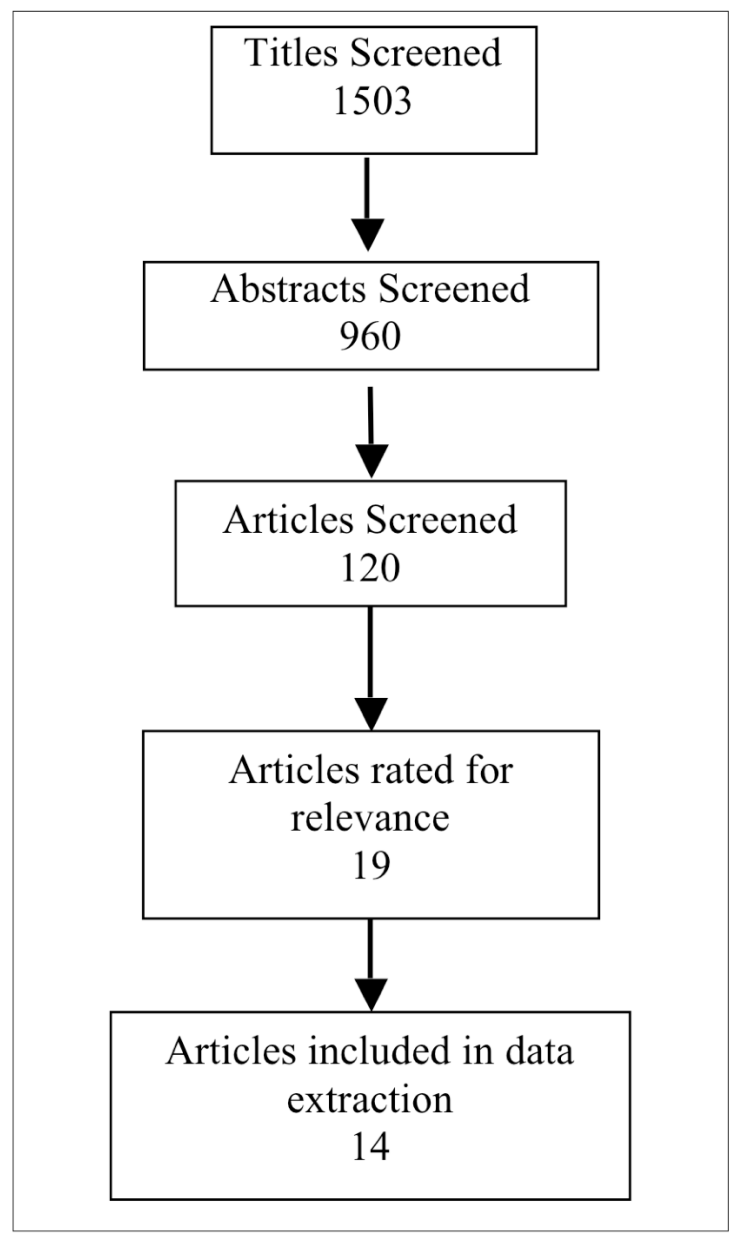

Fig. (1). Search, screening and appraisal results.

The screening process followed three stages [39]. First, titles were screened for relevance to the purpose of the review. Abstracts $(n=960)$ of titles deemed to be relevant by two independent reviewers $(\mathrm{KD}, \mathrm{MJ})$ were retrieved and screened for relevance based on inclusion/exclusion criteria.
Three reviewers applied the criteria to the first 100 abstracts and an inter-rater reliability rating of 0.83 was attained. Consensus was achieved after discussion. One hundred and twenty articles were retrieved and screened by two researchers $(\mathrm{KD}, \mathrm{MJ})$ using the inclusion/exclusion criteria and examined for the level of integration interventions. Nineteen studies were found that met all inclusion criteria.

The final stage of screening was for degree of relevance. Whittemore \& Knafl [38] suggest prioritizing the contribution of each report to the final evaluation. This serves to identify work that is less relevant in order to minimize its final role in the results. Each of the 19 included articles were read and independently assessed by two people (WP, DS) using a three point scale $(1=$ high relevance; $2=$ lower relevance; 3 = exclude). Five articles were excluded on this basis, resulting in 14 articles included in the review.

\section{Quality Appraisal}

Each of the included articles was independently reviewed for quality by two reviewers (KD, MJ), and classified as being excellent, having some limitations or having many limitations [39]. Existing quality assessment checklists specific to quantitative, qualitative and mixed method studies were used for this purpose [40]. Quantitative studies were assessed for concealment of allocation; follow up; blinded assessment of primary outcomes; comparability of groups; baseline measurements; reliable primary outcomes measures; protection against contamination using a checklist from the Cochrane Collaboration Effective Practice and Organization of Care Group (EPOC) [41]. Qualitative studies were assessed for clarity of the research question, context and sampling criteria; appropriate design; adequately described data collection; systematic data analysis; clear results and conclusions; clearly stated authors positions and worth to knowledge [42]. Mixed methods research reports were reviewed for intervention type; appropriate data points and statistical tests; valid and reliable outcome measures; protection against bias and completeness of data set [42]. Discrepancies between the quality ratings were discussed and consensus achieved. Given that none of the articles were rated as having many limitations, all 14 articles remained included at this stage.

\section{Data Analysis and Extraction}

Data from each article was extracted and title, author, date, population, health care setting, relevance and quality scores were recorded. Data pertaining to integration interventions as either structural or relational was extracted. Structural interventions are those aiming to influence the structure or practical organization of how agencies worked together, thereby making it easier to provide integrated care [16]. Relational interventions are I-O strategies aiming to improve relationships between individuals that enhance efforts to better integrate preventive services for marginalized populations [16]. This includes interventions that aim to improve the relations between agencies, organizations and/or communities (versus individual practitioners). Notes were kept regarding expected or unexpected findings regarding design of $\mathrm{I}-\mathrm{O}$ integration interventions, particularly in relation to the effect of the structural and relational interventions. 


\section{RESULTS}

Fourteen articles met our inclusion criteria and have been included in this review (see Table 2). Qualitative $(\mathrm{n}=6)$ study methods were used most commonly, followed by quantitative $(n=5)$ and mixed methodologies $(n=3)$. The majority of articles reported on studies or experiences of integration that took place in urban settings in the United States $(n=9)$ and the United Kingdom $(n=2)$. The remaining articles referred to settings in Canada $(n=1)$, Australia $(n=1)$ and Sweden $(n=1)$. Though terminology and definitions varied, all included articles focused on the provision of integrated care for marginalized populations. As identified in Table $\mathbf{2}$, the included studies focused on community-based services such as primary care, health promotion and preventive health services for marginalized populations such as low income pregnant mothers and their children, persons living with mental illness, at-risk lowincome residents and frail elders.

\section{Quality Appraisal Ratings}

The majority of articles were rated as having minor limitations. Areas of concern regarding the qualitative studies included lack of clarity of the research question, the rigor of data analysis and inadequate descriptions of the relevance or implications of the findings. Common limitations of quantitative studies included questions regarding reliability and validity of measures, completeness of the data set and insufficient follow-up to demonstrate impact of the interventions.

\section{Description of Integration Interventions}

We examined I-O integration interventions in relation to whether their purpose was to effect structural or relational change (see Table 3 ). All of the articles $(n=14)$ reported on structural interventions such as use of structured protocols and feedback [43] and geographical matching of services [44]. Fewer studies explicitly reported on relational

Table 2. Settings, Type of Services, Population and Intervention Type

\begin{tabular}{|c|c|c|c|c|c|}
\hline Authors & Setting & Type of Services & Study Population & \multicolumn{2}{|c|}{ Intervention Type } \\
\hline & & & & Structural & Relational \\
\hline \multicolumn{6}{|c|}{ Qualitative Research Reports } \\
\hline Dea $[52]$ & $\begin{array}{l}\text { Northern } \\
\text { California, } \\
\text { U.S.A. }\end{array}$ & Primary health care & $\begin{array}{c}\text { Primary health care team incorporating } \\
\text { mental heath (physicians, NPs, RNs, } \\
\text { nurse manager) }\end{array}$ & $\mathrm{X}$ & $\mathrm{X}$ \\
\hline Ervin [53] & $\begin{array}{l}\text { New York, } \\
\text { U.S.A. }\end{array}$ & $\begin{array}{l}\text { Multiple health and social } \\
\text { services }\end{array}$ & $\begin{array}{c}\text { Families using } \geq 3 \text { community-based } \\
\text { services }\end{array}$ & $\mathrm{X}$ & \\
\hline $\begin{array}{l}\text { Falk \& Allebeck } \\
\qquad[54]\end{array}$ & $\begin{array}{l}\text { Goteborg, } \\
\text { Sweden }\end{array}$ & $\begin{array}{l}\text { Mental health and social } \\
\text { services }\end{array}$ & Persons living with schizophrenia & $\mathrm{X}$ & $\mathrm{X}$ \\
\hline Gask [50] & U.S.A. & $\begin{array}{l}\text { Primary and specialist mental } \\
\text { health care }\end{array}$ & $\begin{array}{c}\text { Primary and specialist mental health } \\
\text { professionals }\end{array}$ & $\mathrm{X}$ & \\
\hline Rees et al. [45] & Scotland, U.K. & Health and social care services & $\begin{array}{l}\text { Adults with severe and/or enduring } \\
\text { mental health problems }\end{array}$ & $\mathrm{X}$ & \\
\hline Schulz et al. [48] & Detroit, U.S.A. & $\begin{array}{l}\text { Community based organizations, } \\
\text { public health, academic } \\
\text { institution }\end{array}$ & Residents of Detroit's East Side & $\mathrm{X}$ & $\mathrm{X}$ \\
\hline \multicolumn{6}{|c|}{ Quantitative Research Reports } \\
\hline Counsell et al. [55] & Indiana, U.S.A. & $\begin{array}{l}\text { Primary care, public health, } \\
\text { social care and mental health }\end{array}$ & Low income seniors & $\mathrm{X}$ & \\
\hline Harmon et al. [47] & $\begin{array}{c}\text { New South } \\
\text { Wales, Australia }\end{array}$ & $\begin{array}{l}\text { Mental health services \& } \\
\text { physician general practices }\end{array}$ & $\begin{array}{l}\text { Community mental health patients, } \\
\text { nurses, GPs and psychiatrists }\end{array}$ & $\mathrm{X}$ & $\mathrm{X}$ \\
\hline Lyons et al. [46] & Ohio, U.S.A. & $\begin{array}{l}\text { Public health and Emergency } \\
\text { departments }\end{array}$ & $\begin{array}{c}\text { At risk populations needing public health } \\
\text { services }\end{array}$ & $\mathrm{X}$ & \\
\hline Margolis et al. [43] & $\begin{array}{l}\text { North Carolina, } \\
\text { U.S.A. }\end{array}$ & $\begin{array}{l}\text { Primary care, public health, } \\
\text { mental health }\end{array}$ & Low-income pregnant mothers \& infants & $\mathrm{X}$ & $\mathrm{X}$ \\
\hline Tandon et al. [56] & $\begin{array}{l}\text { Baltimore, } \\
\text { U.S.A. }\end{array}$ & Community care and services & $\begin{array}{c}\text { Low income pregnant and parenting } \\
\text { women }\end{array}$ & $\mathrm{X}$ & \\
\hline \multicolumn{6}{|c|}{ Mixed Methods Research Reports } \\
\hline Drennan et al. [51] & England, U.K. & $\begin{array}{l}\text { Primary care, specialist health } \\
\text { and social care }\end{array}$ & 'At risk' elderly living in inner city & $\mathrm{X}$ & \\
\hline Jandorf et al. [13] & $\begin{array}{l}\text { East Harlem, } \\
\text { U.S.A. }\end{array}$ & $\begin{array}{l}\text { Medical center, public hospital } \\
\text { and community health centers }\end{array}$ & At-risk residents of East Harlem & $\mathrm{X}$ & $\mathrm{X}$ \\
\hline Korabek et al. [44] & Canada & $\begin{array}{c}\text { Primary care and home health } \\
\text { care }\end{array}$ & At-risk community-dwelling seniors & $\mathrm{X}$ & $\mathrm{X}$ \\
\hline
\end{tabular}


interventions $(\mathrm{n}=7)$, though several emphasized an implicit relational effect in their results and discussion $(n=4)$.

\section{Structural Interventions}

We identified four categories of I-O interventions that fit with our definition of structural interventions: shared leadership, shared review or development of policies/protocols, changes to referral mechanisms and geographical/caseload matching. A description of each of these categories and examples of interventions are briefly described below.

\section{Shared Leadership}

Shared leadership refers to the presence of established planning bodies with representation from the agencies and/or communities involved. Shared leadership and planning was commonly described as essential to successful I-O integration. Examples of structural entities that facilitated shared planning included steering groups and advisory boards. Key elements attributed to the effectiveness of these I-O bodies included a mission statement or a similar statement of shared objectives and membership that included "...managers with authority to act within their own agencies" [44].

Several authors noted that the implementation of integration activities from a perceived need 'on the ground' is essential to sustainability of shared leadership initiatives [43]. While governmental priorities likely contributed to the establishment of shared leadership, implementation of effective steering groups was the result of synergy between agency-identified need and government backing for improved service integration [43]. Therefore, the need for integration had to be identified and desired at the community level, but was more likely to succeed when also supported by

Table 3. Types of Inter-Organizational Integration Interventions by Article

\begin{tabular}{|c|c|c|c|c|c|c|c|c|c|}
\hline \multirow[t]{2}{*}{ Authors } & \multicolumn{4}{|c|}{ Structural Factors } & \multicolumn{4}{|c|}{ Relational Factors } & \multirow[b]{2}{*}{ 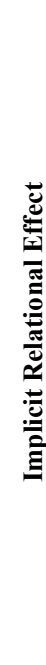 } \\
\hline & 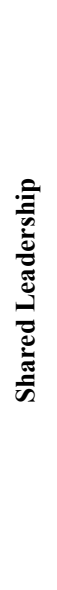 & 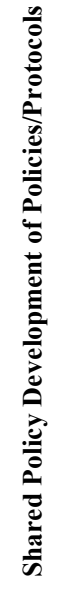 & 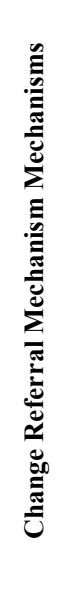 & 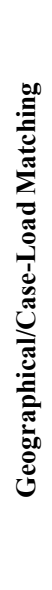 & 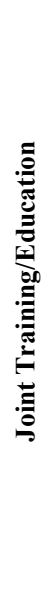 & 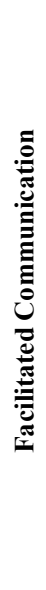 & 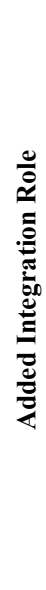 & 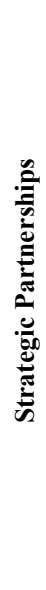 & \\
\hline \multicolumn{10}{|c|}{ Qualitative Research Reports } \\
\hline Dea [52] & & $\mathrm{X}$ & $\mathrm{X}$ & & & & & & \\
\hline \multicolumn{10}{|l|}{ Ervin [53] } \\
\hline Falk \& Allebeck [54] & & & & & & $\mathrm{X}$ & & & \\
\hline Gask [50] & & & & & & & & & $\mathrm{X}$ \\
\hline Rees et al. [45] & & $\mathrm{X}$ & $\mathrm{X}$ & & & & & & \\
\hline Schulz et al. [48] & & & & & $\mathrm{X}$ & & $\mathrm{X}$ & & $\mathrm{X}$ \\
\hline \multicolumn{10}{|c|}{ Quantitative Research Reports } \\
\hline Counsell et al. [55] & & $\mathrm{X}$ & $\mathrm{X}$ & & & & & & \\
\hline Harmon et al. [47] & & $\mathrm{X}$ & $\mathrm{X}$ & $\mathrm{X}$ & & $\mathrm{X}$ & $\mathrm{X}$ & $\mathrm{X}$ & \\
\hline Lyons et al. [46] & & & & $\mathrm{X}$ & & & & & $\mathrm{X}$ \\
\hline Margolis et al. [43] & $\mathrm{X}$ & $\mathrm{X}$ & & & $\mathrm{X}$ & $\mathrm{X}$ & $\mathrm{X}$ & & \\
\hline Tandon et al. [56] & & $\mathrm{X}$ & $\mathrm{X}$ & & & & & & \\
\hline \multicolumn{10}{|c|}{ Mixed Methods Research Reports } \\
\hline Drennan et al. [51] & & & & & & & $\mathrm{X}$ & & $\mathrm{X}$ \\
\hline Jandorf et al. [13] & $\mathrm{X}$ & & $\mathrm{X}$ & & $\mathrm{X}$ & & $\mathrm{X}$ & & \\
\hline Korabek et al. [44] & $\mathrm{X}$ & $\mathrm{X}$ & & $\mathrm{X}$ & $\mathrm{X}$ & $\mathrm{X}$ & & & \\
\hline
\end{tabular}


governmental priorities. For example, Margolis et al.'s [43] multi-level integration interventions targeting state, county and community-level organizations and departments were designed based on this premise.

\section{Shared Review or Development of Policies/Protocols}

Inter-organizational integration was commonly initiated by the recognition that there was a problem with current practice that led to shared review of existing protocols. However, Rees et al. [45] report that the implementation of a new protocol (integrated care pathways) by itself was insufficient to promote integration. Additional interventions, targeted at multiple levels of the system, were recommended to facilitate acceptance and usability of the intervention in practice [45].

\section{Change to Referral Mechanisms}

Structural changes made to improve interagency referral processes were noted to facilitate integration of services. Rees et al. [45] examined the use of integrated care pathways as a means to formalize interagency collaboration between primary care specialists in mental health. Despite reported satisfaction, it was found that the pathway was not being implemented correctly and that 'true integration' was not achieved. This led to the conclusion that managerial involvement is necessary for successful collaboration.

\section{Geographical/Caseload Matching}

Another category of structural interventions includes those designed to align the caseloads of individual providers/programs with those of other organizations. For example, Lyons et al. [46] described co-location of a public health program within an emergency department to screen at-risk clients. Korabek et al. [44] described a different strategy, where realignment of services for seniors enabled partnerships between specialized home care nurses and general practitioners. Caseload matching involved development of software for shared caseload management and management structures and processes to facilitate collaboration. However, authors point out that relational strategies (e.g. honoring the uniqueness of every collaborator, development of a communication blueprint and ongoing team-building exercises) were critical to success of the structural caseload matching intervention [44].

\section{Relational Interventions}

We included studies that either mentioned or directly explored the need for strategies to improve relational aspects of integrated care. Four types of relational interventions were described in the included articles: joint training/education, facilitated communication, addition of an integration role and strategic partnerships.

\section{Joint Training/Education}

Inter-organizational cooperation to provide joint staff training or continuing education was implemented for one of two purposes: to increase the content knowledge of staff from more than one agency and/or to increase staff's and community's knowledge of each other [47, 48]. Schulz et al. [48] described an eight-week training program where health providers met to discuss community problem-solving skills and resources. Regular meetings were also held between the workers and the project coordinator [48]. Although authors may have described increasing staff knowledge as the sole purpose of joint training, this intervention appeared to have an unintentional or implicit effect on the relationships between staff from different organizations.

\section{Facilitated Communication}

A relational intervention that was implemented is that of 'facilitated communication' between staff from multiple organizations. Facilitated communication interventions aim to enhance ongoing communication between practitioners affiliated with different organizations. For example, Korabek et al. [44] described holding four partnership forums annually to promote discussion and learning among all partners. Similarly, as part of a community-wide set of interventions, Margolis et al. [43] implemented regular meetings between agencies and primary care physician practices.

\section{Addition of an Integration Role}

A commonly implemented relational intervention was the addition of a new role with the purpose of facilitating I-O integration. In some cases this role is played by an individual and in other situations an interdisciplinary team may take the responsibility of integrating existing services [13, 47]. For example, Harmon et al. [47] introduced a service model whereby community-based mental health nurses facilitated liaisons between general practitioners and mental health services.

Although the addition of a new role could be described as a structural intervention, the purpose is often to create relationships with the community and the goal is to improve access for marginalized sub-groups within the community. An example of a liaison role is described by Margolis et al. [43] who described the effectiveness of family home visitors as a means to identify and communicate with specific primary care nurses who shared their community based clientele.

\section{Strategic Partnerships}

Strategic partnerships refer to the action of identifying and partnering with organizations based on their established relationship with the community of interest. For example, in the study by Harmon et al. [47], mental health nurses with established connections to other services were selected as the liaison between general practitioners and mental health services. Other authors have described this notion of strategic partnering using less explicit methods, but nevertheless with similar goals and outcomes. For example, Barry \& Britt [49] have described a strategic partnership using a three step process to identify community-based agencies that are "high contact/low or no coercion" as potential partners that would maximize their outreach to atrisk women.

\section{Implicit Relational Effect}

Several studies concluded that interventions had had an important influence on relationships, though this was not their objective. In our analysis, these interventions were labeled as having an implicit relational effect. For example, Gask [50] commented that the "nature and quality of interprofessional conversation" is possibly an "important 
mediating factor in addressing covert barriers to integration". This mediating effect or implicit relationship benefit was also hypothesized to address other types of barriers to the development of effective working relationships, such as insufficient contact between workers and differing views among interdisciplinary staff. These implicit relational effects included facilitating "actual contact" between various groups of workers which then facilitated quality communication between workers [50], close collaboration between specialists [46] and improved teamwork [51].

\section{DISCUSSION}

Despite a large number of articles that focused on service integration, few studies have examined both structural and relational integration interventions to improve access and use of preventive services for marginalized populations. In addition, many of the studies included in this review were qualitative in nature, providing rich understanding of underlying issues, but not enabling examination and comparison of intervention outcomes. To address this gap, we focus our discussion on three issues that should be addressed in future studies of I-O system integration to improve access to preventive services for marginalized populations. The focus on relational as well as structural interventions addresses marginalized clients experiences accessing non-urgent preventive services. The intent is to better understand I-O interventions that may increase use of preventive services focused on improving early childhood development and outcomes. The limitations of this review are also described.

\section{The Imbalance between Effectiveness, Efficiency and Equity}

Our search of the literature revealed a relative imbalance between studies focused on interventions to improve effectiveness and efficiency of services versus attempts to improve equitable access to services. This echoes the findings of a review of integrated perinatal care by Rodriguez \& Rivières-Pigeon [17]. The emphasis on effectiveness and efficiency at the oversight of equity means that existing population health inequities will persist, in spite of efforts to better integrate care across organizations. We propose that a population health orientation would add equity to the more common efficiency and/or effectiveness aims of integration. In addition, very few research articles explored the impact of I-O interventions from a client perspective. There is a need for greater systematic inquiry into client perspectives on how I-O integration interventions impact their experiences of care. Adding this population health perspective would facilitate examination of the difference in effectiveness across populations, and enable organizations to more closely address the experiences of marginalized families, thereby capitalizing on the life-long impacts of improving early childhood developmental outcomes.

\section{Increase Visibility of Relational Integration}

A second gap in the research literature relates to the 'invisibility' of the relational dimension of service integration. While an implicit relational effect was noted as key to the success of structural interventions, it was often not an explicit aim of the intervention. This represents a conceptual as well as operational gap in knowledge related to I-O interventions to integrate care. Greater attention to the relational dimension of services could focus efforts on improving how marginalized families experience care and enable providers to share experiences and strategies for improving access and participation in preventive services. Categorizing integration interventions as relational or structural may aid in understanding the sequencing, combination and conditions required for integration interventions to improve access to care. It is a method for recognizing and strategically addressing relational and structural changes that are needed to move from historically separated organizations and programs to a more equitable system of integrated care. Greater clarity around the concept of relational interventions and the level of relationships involved (e.g. staff-community, provider-provider) is needed. More research is required regarding the design and testing of interventions at the I-O level to improve the relational aspects of service integration that may improve clients' experiences accessing non-urgent preventive services during the early childhood period, and ultimately reduce health disparities.

\section{Need for Multiple Interventions}

Our findings suggest that one-off structural interventions are not enough to impact I-O integration. To overcome system barriers to integration, it is essential to include relational interventions that support structural changes at the organizational and clinical care levels $[45,50]$. More studies aiming to strategically combine and test multiple interventions that explicitly focus on improving relational as well as structural dimensions of integration are needed, with particular attention to the impact on reducing disparities in access to non-urgent preventive services.

Furthermore, several authors describe the need for multiple interventions strategically targeted at different levels of the service system. Specifically, the need for involvement at the managerial (or higher) level of organizations must accompany interventions to improve integration at the individual practitioner level [45]. Commitment to, and involvement in integration interventions at the higher administrative and governance levels of service provision is needed if interventions at other levels are to succeed $[45,46]$. These findings suggest the need for the design of interventions targeted at changing funding structures and the culture of practice to overcome system barriers to service integration. To reduce inequities experienced by marginalized families, population health policy makers need accounts of theoretically based I-O integration interventions that include reporting on the intervention model, study design, integrity of intervention/process evaluation, particulars of the context, and the differential effects across populations [30, 31]. Commitment and action at these levels will be more likely to achieve and sustain changes required for equitable access to preventive services for marginalized families.

\section{Limitations}

The search was limited to articles from six databases published since 2000 and did not include articles in countries other than those we identified as having similar demographic and service contexts to Canada. Though this enhances 
generalizability and fittingness to Canadian contexts, these methodological decisions may have excluded other informative studies. Lack of conceptual clarity in the literature regarding such terms as 'service integration' and 'marginalized populations' created challenges for developing an effective search strategy. As a result, we used an iterative cycle of screening decisions related to the variation in defining the concepts. These decisions were made explicit in our methods. Finally, the benefits of the inclusive nature of integrative review methods resulted in developing rich knowledge based on several qualitative studies in concert with quantitative and mixed methods studies. However, the integrative review method did not enable comparison or comment on outcomes, as is expected in the systematic review method.

\section{CONCLUSION}

Results of this integrative review add to the theory and logic that may inform design of inter-organizational interventions to better integrate services to improve access to preventive services for marginalized families. Specifically, we devised an approach to more explicitly examine structural and relational impacts found in research studies aiming to improving I-O integration and access to preventive services for marginalized families. Though there is a small amount of research on this topic, systematic inquiry into the effectiveness of system integration interventions in improving access to preventive services for marginalized families is required. Furthermore, the literature on service integration has been dominated by investigation into the structural dimensions of I-O integration. Although this work has outlined key structural factors that can be addressed in interventions to improve service delivery, relational dimensions of the process of service integration have been few in comparison. Relational factors likely play a key role in improving access to care for marginalized families across the broad spectrum of organizations involved in providing non-urgent health-promoting and preventive care. It appears that further investigation regarding the importance of the relational effect of integration interventions is particularly important when trying to improve access of marginalized families to non-urgent care, to understand their experience beyond that of any client group seeking any type of care (i.e. not necessarily preventive care). Future study regarding I-O integration should include the design of interventions that address both structural and relational barriers to integration, and explicate differential influence on marginalized families' access to non-urgent preventive services.

\section{ACKNOWLEDGEMENTS}

We gratefully acknowledge funding support for this work from the Community Health Research Unit, Ontario Ministry of Health and Long Term Care. We thank Wendy Gifford for reviewing earlier versions of this manuscript. The team also wishes to acknowledge the Growing Healthy: Connecting services to families with young children initiative in Ottawa, Ontario for inspiring us to conduct this review.

\section{REFERENCES}

[1] Hertzman C. The case for an early childhood development strategy. ISUMA. Can J Policy Res 2000; 1(2): 1-17.
[2] Irwin LG. Preliminary review of the evidence base for healthy infant and early childhood development in BC; Healthy Child BC Forum 2004

[3] Affonso D, Mayberry L, Inaba A, Matsuno R, Robinson E. Hawaiian-style Talkstory: psychosocial assessment and intervention during and after pregnancy. J Obstet Gynecol Neonatal 1996; 25: 737-42.

[4] Bucharski D, Brockman L, Lambert D. Developing culturally appropriate prenatal care models for Aboriginal women. Can J Hum Sex 1999; 34(1): 151-4.

[5] Fisher P, Ball T. The Indian family wellness project: an application of the tribal participatory research model. Prev Sci 2002; 3(3): 23540.

[6] Martens P. Increasing breastfeeding initiation and duration at a community level: an evaluation of Sagkeeng First Nation's community health nurse and peer counselor programs. J Hum Lact 2002; 18(3): 236-46.

[7] Smith D, Edwards N, Varcoe C, Martens P, Davies B. 'Making a Difference': a new care paradigm for pregnant and parenting aboriginal people. Can J Public Health 2007; 98(4): 321-4.

[8] Olds DL, Sadler L, Kitzman H. Programs for parents of infants and toddlers: recent evidence from randomized trials. J Child Psychol Psychiatr 2007; 48(3-4): 355-91.

[9] Sanders MR, Prinz RJ, Shapiro CJ. Predicting utilization of evidence-based parenting interventions with organizational, service-provider and client variables. Adm Policy Ment Health 2009; 36(2): 133-43. Epub 2009 Feb 13.

[10] Asthana S, Halliday J. What can rural agencies do to address the additional costs of rural services? A typography of rural service innovation. Health Soc Care Community 2004; 2(6): 457-65.

[11] Canadian Council on Social Development; The progress of Canada's children, $7^{\text {th }}$ ed. Ottawa: Author. 2007. [cited: 2007 June 16]. Available from: http://www.ccsd.ca/pccy/2006

[12] Moghadam E, Peterson W, Hanvey L, Birmingham M, Smith D. Integrating care for Vulnerable Families. In: Proceedings of the 2006 Provincial Conference of the Ontario Public Health Association. Cornwall, Ontario: Ontario Public Health Association 2006; p. 19.

[13] Jandorf L, Fatone A, Borker PV, et al. Creating alliances to improve cancer prevention and detection among urban medically underserved minority groups: The East Harlem partnership for cancer awareness. Can Suppl 2006; 107(8): 2043-51.

[14] Tunstill J, Allnock D. Understanding the contribution of sure start local programmes to the task of safeguarding children's welfare. The National Evaluation of Sure Start Team, Birkbeck College, University of London. 2007. [cited: 2009 April 27]. Available from: http://www.surestart.gov.uk/doc/P0002466.pdf

[15] New Zealand Ministry of Social Development. Review of strengthening families local collaboration. 2005. [cited: 2009 April 27]. Available from: http://www.strengtheningfamilies.govt.nz/doc uments/resources/reports/review-of-strengthening-families-local-co llaboration.doc

[16] Park J, Turnbull AP. Service integration in early intervention: Determining interpersonal and structural factors for its success. Infants Young Child 2003; 16(1): 48-58.

[17] Rodríguez C, Rivières-Pigeon C. A literature review on integrated perinatal care. Int J Integr Care 2007; 7: E28.

[18] Browne G, Roberts J, Gafni A, Byrne C, Kertyzia J, Loney P. Conceptualizing and validating the human services integration measure. Int J Integr Care [serial on the internet]. 2004. [cited: July 31, 2007]. Available from: http://www.ijic.org

[19] Bronfenbrenner U, Evans G. Developmental science in the $21^{\text {st }}$ Century: emerging questions, theoretical models, research designs and empirical findings. Soc Dev 2000; 9: 115-25.

[20] Kodner DL, Spreeuwenberg C. Integrated care: Meaning, logic, applications, and implications - a discussion paper. Int J Integr Care [serial on the internet] 2002; Available from: http://www.ijic.org

[21] Edwards N, Mill J, Kothari AR. Multiple intervention research programs in community health. Can J Nurs Res 2004; 36(1): 40-54.

[22] Haggerty JL, Reid RJ, Freeman GK, Starfield BH, Adair CE, McKendry R. Continuity of care: a multidisciplinary review. $\mathrm{Br}$ Med J 2003; 327: 1219-21.

[23] Sheikh-Mohammed M, Macintyre CR, Wood NJ, Leask J, Isaacs D. Barriers to access to health care for newly resettled sub-Saharan refugees in Australia. Med J Aust 2006; 185(11-12): 594-7. 
[24] Guendelman S, Angulo V, Oman D. Access to health care for children and adolescents in working poor families: recent findings from California. Med Care 2005; 43(1): 68-78.

[25] Sword W. A socio-ecological approach to understanding barriers to prenatal care for women of low income. J Adv Nurs 1999; 29(5): $1170-7$.

[26] Peterson WE, Sword W, Charles C, DiCenso A. Adolescent's perceptions of inpatient postpartum nursing care. Qual Health Res 2007; 17(2): 201-12.

[27] Ruddick L. Health of people with intellectual disabilities: a review of factors influencing access to health care. Br J Health Psychol 2005; 10(4): 559-70.

[28] Hall J. Marginalization revisited: critical, postmodern, and liberation perspectives. Adv Nurs Sci 1999; 22: 88-102.

[29] Lynam J, Cowley S. Understanding marginalization as a social determinant of health. Crit Public Health 2007; 17(2): 137-49.

[30] Rychetnik L, Frommer M, Hawe P, Shiell A. Criteria for evaluating evidence on public health interventions. J Epidemiol Community 2002; 56: 119-27.

[31] Armstrong R, Waters E, Moore L, et al. Improving the reporting of public health intervention research: advancing TREND and CONSORT. J Public Health 2008; 30(1): 103-9.

[32] Olds D. Prenatal and infancy home visiting by nurses from randomized trials to community replication. Prev Sci 2002; 3(3): $153-72$.

[33] Martin-Meisner R, Valaitis R. A scoping literature review of collaboration between primary care and public health. A report to the Canadian Health Services Research Foundation. Hamilton, Ontario. 2008 [cited: 2009 March 20]. Available from: http://fhs. mcmaster.ca/nursing/docs/MartinMisener-Valaitis-Review.pdf

[34] Drummond JE, Weir AE, Kysela GM. Home visitation practice: models, documentation and evaluation. Public Health Nurs 2002; 19(1): 21-9.

[35] Nicholls C. Role redesign to support the delivery of integrated care. J Commun Nurs 2005; 19(12): 4-8.

[36] Mulrow CD. Systematic reviews: rationale for systematic reviews. Br Med J 1994; 309: 597-9.

[37] Mays N, Pope C, Popay J. Details of approaches to synthesis. A methodological appendix to the paper: systematically reviewing qualitative and quantitative evidence to inform management and policy making in the health field. J Health Serv Res Policy 2005; 3(Suppl 1): 6-20.

[38] Whittemore R, Knafl K. The integrative review: updated methodology. J Adv Nurs 2005; 52(5): 546-53.

[39] Gifford W, Davies B, Edwards N, Griffin P, Lybanon V. Managerial leadership for nurses' use of research evidence: an integrative review of the literature. Worldviews Evid Based Nurs 2007; 4(3): 126-45.

[40] Greenhalgh T, Robert G, Bate P. How to spread good ideas: a systematic review of the literature on diffusion, dissemination and sustainability of innovations in health service delivery and organization; Report for the National Co-ordinating Centre for NHS Service Delivery and Organization R \& D (NCCSDO). 2004. [cited: 2007 January 24]. Available from: http://www.sdo.lshtm. ac.uk/files/project/38-final-report.pdf
[41] Cochrane Effective Practice and Organization of Care Review Group. The data collection check-list 2002 [cited: 2009 April 27]. Available from: www.epoc.cochrane.org/en/index.html

[42] Greenhalgh T, Robert G, MacFarlane F, Bate P, Kyriakidou O. Diffusion of Innovations in Service Organizations: Systematic Review and Recommendations. The Millbank Quarterly: A Multidisciplin J Popul Health Health Policy [serial on the internet] 2004; 82. [cited: 2007 July 22]. 4; [about 20 screens] Available from: http://www.milbank.org/quarterly/8204feat.html

[43] Margolis P, Stevens R, Bordley C, et al. From concept to application: the impact of community-wide intervention to improve the delivery of preventive services to children. Pediatrics 2001; 108(3): E42.

[44] Korabek B, Rosenau P, Slauenwhite C, Ross L. Home care/physician partnerships in the community: a Canadian model in development. Home Health Care Manage Pract 2004; 16(4): 261-8.

[45] Rees G, Huby G, McDade L, McKechnie L. Joint working in community mental health teams: implementation of an integrated care pathway. Health Soc Care Community 2004; 12(6): 527-36.

[46] Lyons MS, Lindsell CJ, Ledyard HK, Frame PT, Trott AT. Health departmental collaboration with emergency departments as a model for public health programs among at-risk populations. Public Health Rep 2005; 20(3): 259-65.

[47] Harmon K, Carr VJ, Lewin TJ. Comparison of integrated and consultation-liaison models for providing mental health care in general practice in New South Wales, Australia. J Adv Nurs 2000; 32(6): 1459-66.

[48] Schultz AJ, Israel BA, Parker EA, Lockett M, Hill Y, Wills R. The East-side village health worker partnership: integrating research with action to reduce health disparities. Public Health Rep 2001; 116: 548-55

[49] Barry K, Britt DW. Outreach: targeting high-risk women through community partnerships. Women's Health Issues 2002; 12(2): 6678.

[50] Gask L. Overt and covert barriers to the integration of primary and specialist mental health care. Soc Sci Med 2005; 6(8): 1785-94.

[51] Drennan V, Iliffe S, Haworth D, Tai SS, Lenihan P, Deave T. The feasibility and acceptability of a specialist health and social care team for the promotion of health and independence in 'at risk' older adults'. Health Soc Care Community 2005; 13(2): 136-44.

[52] Dea RA. The integration of primary care and behavioral; healthcare in Northern California Kaiser-Permanente. Psychiatr Q 2000; 71(1): 17-29.

[53] Ervin NE. Assessing interagency collaboration through perceptions of families. J Community Health Nurs 2004; 21(1): 49-60.

[54] Falk K, Allebeck P. Implementing assertive community care for patients with schizophrenia: a case study of co-operation and collaboration between mental health care and social services. Scand J Caring Sci 2002; 16: 280-6.

[55] Counsell SR, Callahan CM, Buttar AB, Clark DO, Frank KI. Geriatric resources for assessment and care of elders (GRACE): a new model of primary care for low-income senior. J Am Soc Geriatr Dent 2006; 54: 1136-41

[56] Tandon D, Parillo K, Jenkins C, Jenkins J. Promotion of service integration among home visiting programs and community coalitions working with low income pregnant and parenting women. Health Promot Pract 2007; 8(1): 79-87. 